\title{
Prevalencia de síndrome metabólico en población adulta chilena: Datos de la Encuesta Nacional de Salud 2003
}

\author{
ANDREA A. VALENZUELA B. ${ }^{1}$, ALBERTO MAÍZ1', \\ PAULA MARGOZZINI ${ }^{2}$, CATTERINA FERRECCIO ${ }^{2}$, ATTILIO RIGOTTI ${ }^{3}$, \\ RICARDO OLEA ${ }^{4}$, ANTONIO ARTEAGA ${ }^{1}$
}

Background: There are several diagnostic criteria for Metabolic Syndrome (MS) definition. Aim: To study their application in the Chilean general adult population. Material and Methods: We analyzed data from a random sub sample of 1.833 adults aged 17 years and older surveyed during the First Chilean National Health Survey conducted in 2003. The prevalence of MS was estimated using the update Adult Treatment Panel III (ATP III) of the National Cholesterol Education Program (NCEP) and the International Diabetes Federation (IDF 2005) criteria. The distribution of MS was analyzed according to age, gender, educational level, geographic area, obesity and sedentary lifestyle. Results: The overall prevalence of MS was 31.6\% (95\% CI 28.5-34.9) and 36.8\% (95\% CI 33.5-40.3), according to update ATPIII-NCEP and IDF criteria respectively. Both criteria had a 90\% concordance. Demographic and socioeconomic distribution was similar for both criteria. The prevalence of high blood pressure, high fasting glucose, and low HDL cholesterol (MS components) were: 46, 22 and 53\% respectively. The prevalence of abnormal waist circumference was 30 and 59\% according to update ATPIII-NCEP and IDF criteria, respectively. Using update ATPIII-NCEP criteria, the gender, age and educational level adjusted odds ratio (OR) for having MS was 9.59 (95\% IC 6.8 13.6) for obese subjects compared with normal weight subjects and 2.14 (95\% IC 1.3 3.7) for sedentary subjects compared with non sedentary. Conclusions: There was a 90\% agreement between update ATPIII-NCEP and IDF criteria for the diagnosis of MS. The overall prevalence of MS in this population was 32\% usuing update ATPIII-NCEP criteria, with higher prevalence among obese and sedentary subjects.

(Rev Med Chile 2010; 138: 707-714).

Key words: Hyperinsulinism; Metabolic syndrome X; Prevalence.

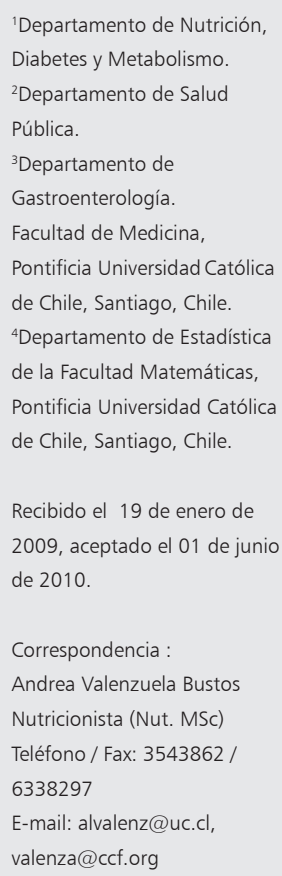

4 n 1988, Reaven definió el "Síndrome X" como la asociación de diferentes elementos clínicos y de laboratorio en un individuo: hipertensión arterial (HTA), intolerancia a la glucosa, elevación de triglicéridos (TGS) y reducción del colesterol HDL (cHDL), señalando que estos atributos se asociarían más allá del azar con un nexo fisiopatológico común: resistencia insulínica $(\mathrm{RI})^{1}$. Esta condición conlleva un mayor riesgo de diabetes mellitus tipo 2 (DM2) y enfermedades cardiovasculares $(\mathrm{ECV})^{2-4}$.

La etiopatogenia del SM no esta aún aclarada y, no existe acuerdo acerca del nexo común de sus componentes. Se ha destacado el rol de la obesidad 
abdominal (OA), la que se ha planteado como elemento fisiopatológico común y esencial ${ }^{5,6}$. La RI no está presente en la totalidad de los casos ni se ha logrado explicar su rol en la etiopatogenia del SM. Esto, ha llevado a importantes divergencias y controversias en los criterios recomendados para el diagnóstico del SM.

Recientes críticas señalan que la asociación de atributos no tendría un nexo etiopatogénico común único, y que la predicción del riesgo de DM2 y ECV derivada de la identificación del SM, no sería mayor que la suma de sus componentes individuales por lo que su diagnóstico no tendría utilidad clínica ${ }^{7}$.

Pese a reconocer algunas de las críticas anteriores, la Organización Mundial de Salud (OMS), American Heart Association, National cholesterol Education Program Adult Treatment Panel III (NCEP ATPIII) y Federación Internacional de Diabetes (IDF), aceptaron la utilidad clínica del concepto de SM ya que estudios prospectivos han establecido su rol predictivo significativo e independiente para DM2 y ECV ${ }^{8-10}$.

En el año 2001 NCEP ATPIII considera al SM como una entidad definida que confiere mayor riesgo cardiovascular ${ }^{11}$. En este criterio, la simple presencia de 3 de los 5 factores clínicos o de laboratorio (en cualquier combinación) constituye la base para establecer el diagnóstico. A diferencia del criterio $\mathrm{OMS}^{12}$, NCEP ATPIII no exige demostrar directamente presencia de RI (mediciones directas son laboriosas y no bien estandardizadas para uso rutinario). Además, la definición del NCEP ATPIII sería de fácil aplicación en la práctica clínica, siendo suficiente la simple medición de la circunferencia de cintura (CC).

Los puntos de corte de CC (pCC) provienen de las Guías Clínicas para el Diagnóstico y Manejo de Obesidad ${ }^{13}$ y fueron definidos en base a los estudios de Lean ME. y Han TS, quienes establecieron que los $\mathrm{pCC} \geq 94 \mathrm{~cm}$ para hombres $\mathrm{y} \geq 80 \mathrm{~cm}$ para mujeres identifican a sujetos con IMC $\geq 25$, mientras que los pCC $\geq 102 \mathrm{~cm}$ en hombres $\mathrm{y} \geq 88 \mathrm{~cm}$ en mujeres identifican a sujetos con IMC $\geq 30^{14-16}$.

En el año 2005 se publicó la actualización del criterio NCEP ATPIII (ATPIII actualizado) ${ }^{17}$, donde además de reducir el umbral de glicemia de ayuno a $100 \mathrm{mg} / \mathrm{dl}^{18}$, se incluyó aquellos pacientes con tratamiento farmacológico para DLP, HTA e intolerancia a la glucosa o DM2.

En el 2005, la Federación Internacional de Diabetes (IDF) ${ }^{19}$ publicó sus criterios para definición de
SM, acogieron favorablemente la definición ATPIII actualizada por su simplicidad clínica y utilidad práctica, pero consideraron como principal criterio para el diagnóstico del SM la OA. La presencia de esta condición asociada a 2 de los factores originales del criterio ATP III actualizado, serían suficientes para el diagnóstico de SM. Además, la IDF estableció que un IMC de $25 \mathrm{~kg} / \mathrm{m}^{2}$ sería el punto de corte para definir el valor de CC que se utilizaría como criterio diagnóstico de OA. Así, para la población Latina se sugiere utilizar los pCC correspondientes al Sudeste Asiático, $\geq 90 \mathrm{~cm}$ en hombres y $\geq 80 \mathrm{~cm}$ en mujeres ${ }^{14,20}$. IDF estima que los pCC deberían ser determinados a través de estudios epidemiológicos en cada población de interés.

La Encuesta Nacional de Salud (ENS) efectuada en Chile por el Ministerio de Salud (MINSAL) el año $2003^{21}$, refleja que aproximadamente el $60 \%$ de la población presenta sobrepeso u obesidad y que el $22,6 \%$ presenta SM diagnosticado con el criterio NCEP ATP III 2001. La elevada prevalencia de SM y su condición de factor de riesgo independiente de DM2 y ECV aconsejan uniformar su definición clínica, permitiendo al equipo de salud incorporar este concepto a estrategias individuales y poblacionales para la prevención de DM2 y ECV.

El objetivo de este trabajo fue analizar el grado de concordancia en la prevalencia de SM en la población adulta según la aplicación de los criterios ATPIII actualizado e IDF y establecer la asociación del SM con género, edad, ruralidad, nivel educacional, obesidad y sedentarismo en Chile.

\section{Materiales y Sujetos}

El grupo de población chilena estudiada por la Encuesta Nacional de Salud (ENS) 2003 corresponde a una muestra aleatoria de 3.600 personas, representativa de la población general, de ambos géneros, de residencia rural y urbana, mayor de 17 años, de todo el país. La metodología empleada para la selección de esta muestra poblacional se encuentra descrita en extenso ${ }^{21,22}$.

Los participantes fueron examinados en el hogar por enfermeras entrenadas, quienes evaluaron una serie de parámetros: antropometría, presión arterial y muestra de sangre, entre otros.

Para el análisis de prevalencia de SM, se utilizó una submuestra aleatoria de sujetos $(\mathrm{n}=1.833)$ y se obtuvo una muestra de sangre para análisis de lípidos (ayuno 9 o más horas). Todos los exámenes 
de laboratorio fueron realizados en el Laboratorio Central de la Red de Salud de la Facultad de Medicina de la Pontificia Universidad Católica de Chile. Los lípidos fueron medidos con el método enzimático colorimétrico Hitachi (cálculo colesterol LDL por fórmula de Friedwald) y la glicemia se cuantificó con el método de hexoquinasa. La CC fue medida en la línea media axilar en el punto medio entre el reborde costal y la cresta ilíaca, con huincha plástica no rígida diseñada para el estudio. La presión arterial fue medida utilizando un instrumento automatizado (OMRON-HEM 713C), promediando dos mediciones en la misma visita, separadas por 2 minutos, bajo condiciones de ayuno, horario matinal después de 5 minutos de reposo previo, vaciamiento vesical previo. La obesidad fue definida como un IMC $\geq 30$ y el sedentarismo según la definición disponible en la ENS2003.

En la Tabla 1 se detallan los criterios diagnósticos ATPIII actualizados e IDF utilizados para el estudio.

\section{Estadística}

Fue realizada en concordancia con el muestreo complejo utilizando los factores de expansión de ENS 2003. El cálculo de varianzas, intervalos de confianza y modelos de regresión logística se realizaron utilizando el módulo de muestras complejas del paquete SPSS 13.0. La concordancia de diagnóstico de SM entre los 2 criterios clínicos utilizados se analizó aplicando el test Kappa.

\section{Resultados}

En la ENS 2003 la prevalencia de SM fue 22,6\% con el criterio NCEP ATP III 2001. En nuestro estudio la prevalencia de SM fue 31,6\% (IC 95\% 28,5-34,9) para el criterio diagnóstico ATPIII actualizado y $36,8 \%(33,5-40,3)$ para el criterio IDF (Figura 1). La concordancia del diagnóstico entre ambas definiciones fue 90\% (88,1-91,7) y la prevalencia de quienes son clasificados en promedio como individuos que presentan SM con ambas definiciones fue 29,2\% (26,2-32,4). El valor estadístico Kappa fue 0,779, indicando una buena concordancia ${ }^{23}$. Por lo tanto, el 90\% de la población adulta chilena sería clasificada de igual forma con ambos criterios.

Las prevalencias aisladas para cada uno de los componentes de la definición de SM según las diferentes clasificaciones se describen en Tabla 2. La presencia de un nivel de cHDL bajo fue el atributo más frecuente para el diagnóstico de SM en Chile,

Tabla 1. Criterios para el diagnóstico clínico del síndrome metabólico

\begin{tabular}{|c|c|c|}
\hline Criterios diagnóstico & NCEP ATPIII actualizado (2005) & IDF \\
\hline Requisito & 3 de los 5 factores de riesgo siguientes & $\begin{array}{l}\text { Presencia de obesidad central dada por CC } \\
\text { según pertenencia étnica más } 2 \text { de los } 4 \\
\text { factores de riesgo restantes }\end{array}$ \\
\hline $\begin{array}{l}\text { Circunferencia de cintura } \\
(\mathrm{cm})\end{array}$ & $\begin{array}{l}\text { CC: } \\
\text { Hombres: }>102 \\
\text { Mujeres: }>88\end{array}$ & $\begin{array}{l}\text { CC (población específica): } \\
\text { Personas de origen centro o sur americano: } \\
\text { Hombres: } \geq 90 \\
\text { Mujeres: } \geq 80\end{array}$ \\
\hline Triglicéridos (mg/dl) & \multicolumn{2}{|l|}{ TG $\geq 150$ o en tratamiento farmacológico para HTG } \\
\hline Colesterol HDL (mg/dl) & \multicolumn{2}{|l|}{$\begin{array}{l}\text { CHDL: } \\
\text { Hombres: }<40 \\
\text { Mujeres: }<50 \\
\text { o en tratamiento farmacológico para cHDL bajo }\end{array}$} \\
\hline Presión arterial (mmHg) & \multicolumn{2}{|c|}{$\geq 130$ /o $\geq 85$ o tratamiento farmacológico para hipertensión } \\
\hline Glicemia (g/dl) & \multicolumn{2}{|l|}{$\begin{array}{l}\geq 100 \text { (incluidos sujetos con DM2) } \\
\text { o tratamiento farmacológico para glicemia elevada }\end{array}$} \\
\hline
\end{tabular}

NCEP ATP III: National Cholesterol Education Program Adult Panel Treatment. IDF: International Diabetes Federation. DM2: diabetes mellitus tipo 2, CC: circunferencia de cintura, TG: triglicéridos, cHDL: colesterol HDL, HTG: hipertrigliceridemia. 
seguido de la elevación de las cifras de presión arterial. Todos los componentes tenían igual prevalencia con ambos criterios, excepto en CC. La prevalencia de OA aplicando el criterio de ATPIII actualizado fue $29,7 \%$, aumentando prácticamente al doble $59,4 \%$ con el criterio IDF. En el $27 \%$ de la población coexisten al menos tres factores del SM distintos a OA.

La distribución de SM por edad poblacional fue similar para ambos criterios diagnósticos, mostrando en ambos casos un incremento progresivo con la edad. Así, en menores de 25 años, solamente 7\% (ATPIII actualizado) o 8\% (IDF) de la población presenta SM, después de los 65 años aumenta a 56\% (ATPIII actualizado) o $60 \%$ (IDF), sin diferencias entre hombres y mujeres (Tabla 3 ).

$\mathrm{Al}$ analizar por escolaridad, el nivel bajo presenta mayores prevalencias de SM, 40,4\% y $46,9 \%$
ATPIII actualizado e IDF respectivamente, mientras que en el nivel alto la prevalencia fue $26,1 \%$ y $30,3 \%$ respectivamente. Al analizar por género, la prevalencia de $S M$ en mujeres con bajo nivel de escolaridad alcanza $44,2 \%$ y $49 \%$ para ambos criterios respectivamente. Las mujeres con nivel alto de escolaridad presentan una menor prevalencia (12,4\% ATPIII actualizado y 14,5 IDF). Esta última diferencia es estadísticamente significativa al ajustar por edad.

La prevalencia de SM por regiones mostró importantes variaciones a lo largo del país. En la V Región se observó una prevalencia de $27 \%$ y $32 \%$, lo que contrasta con la I Región que alcanzó valores de $48 \%$ y $52 \%$. Tomando como referencia la Región Metropolitana (RM), las regiones I, IV y VIII presentaron tasas significativamente mayores de SM, incluso después de ajustar por

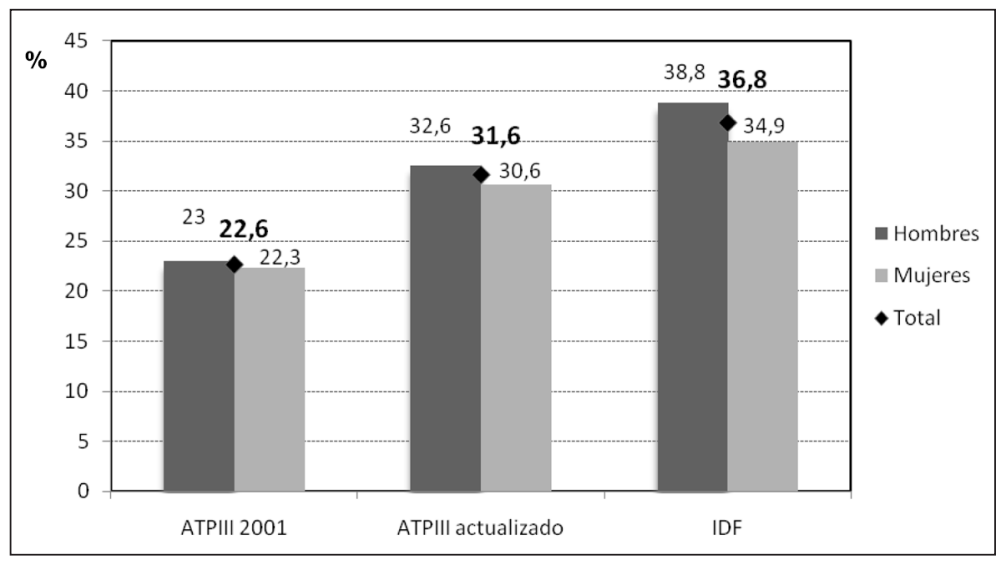

Figura 1. Prevalencia de síndrome metabólico en población general adulta, Chile, ENS 2003.

Tabla 2. Prevalencias aisladas e intervalos de confianza (IC) $95 \%$ de los componentes del
síndrome metabólico en Chile, ENS 2003

\begin{tabular}{|c|c|c|}
\hline Componentes de SM & $\begin{array}{l}\text { Prevalencia (\%) e IC } \\
\text { NCEP ATPIII actualizado }\end{array}$ & $\begin{array}{c}\text { Prevalencia (\%) e IC } \\
\text { según IDF }\end{array}$ \\
\hline Circunferencia de cintura (CC) aumentada & $29,7^{*}(26,6-33,0)$ & $59,4^{* *}(55,7-63,0)$ \\
\hline Presión arterial elevada & $46,0 \quad(42,5-49,6)$ & $46,0 \quad(42,5-49,6)$ \\
\hline Glicemia elevada & $22,0 \quad(19,0-25,4)$ & $22,0 \quad(19,0-25,4)$ \\
\hline Hipertrigliceridemia & $30,0 \quad(27,0-33,2)$ & $30,0 \quad(27,0-33,2)$ \\
\hline Colesterol HDL bajo & $52,6 \quad(48,7-55,6)$ & $52,6 \quad(48,7-55,6)$ \\
\hline
\end{tabular}

*CC: $\geq 102 \mathrm{~cm}$ en hombres $y \geq 88 \mathrm{~cm}$ en mujeres. ** CC: $>90 \mathrm{~cm}$ en hombres y $>80 \mathrm{~cm}$ en mujeres. NCEP ATP III: National Cholesterol Education Program Adult Panel Treatment. IDF: International Diabetes Federation. CC: circunferencia de cintura. 
Prevalencia de síndrome metabólico en adultos chilenos - A. Valenzuela B. et al

Tabla 3. Prevalencia e intervalo de confianza $95 \%$ del síndrome metabólico basado en NCEP ATPIII actualizado según edad y sexo

\begin{tabular}{|c|c|c|c|c|c|c|}
\hline \multirow[t]{2}{*}{ Edad } & \multicolumn{2}{|r|}{ Hombres } & \multicolumn{2}{|r|}{ Mujeres } & \multicolumn{2}{|r|}{ Total } \\
\hline & $\mathbf{n}$ & (IC) & $\mathbf{n}$ & (IC) & $\mathbf{n}$ & (IC) \\
\hline $17-24$ & 102 & $10,6 \quad(4,8-22)$ & 114 & $2,9 \quad(0,9-8,8)$ & 216 & $6,7 \quad(3,4-12,6)$ \\
\hline $25-44$ & 266 & $31,1(24,3-38,7)$ & 282 & $27,7 \quad(21-35,6)$ & 548 & $24,9(24,5-34,9)$ \\
\hline $45-64$ & 263 & $43,9(35,4-52,8)$ & 333 & $44,8 \quad(37-52,9)$ & 596 & $44,4(38,3-50,7)$ \\
\hline $65 y+$ & 202 & $55,1(44,6-65,2)$ & 256 & $(46-65)$ & 458 & $55,5(48,7-62,1)$ \\
\hline Total & 833 & $32,7(28,3-37,5)$ & 985 & $30,8(26,7-35,2)$ & 1.818 & $31,6(28,6-35,1)$ \\
\hline
\end{tabular}

ODDS RATIO corregida por edad y sexo

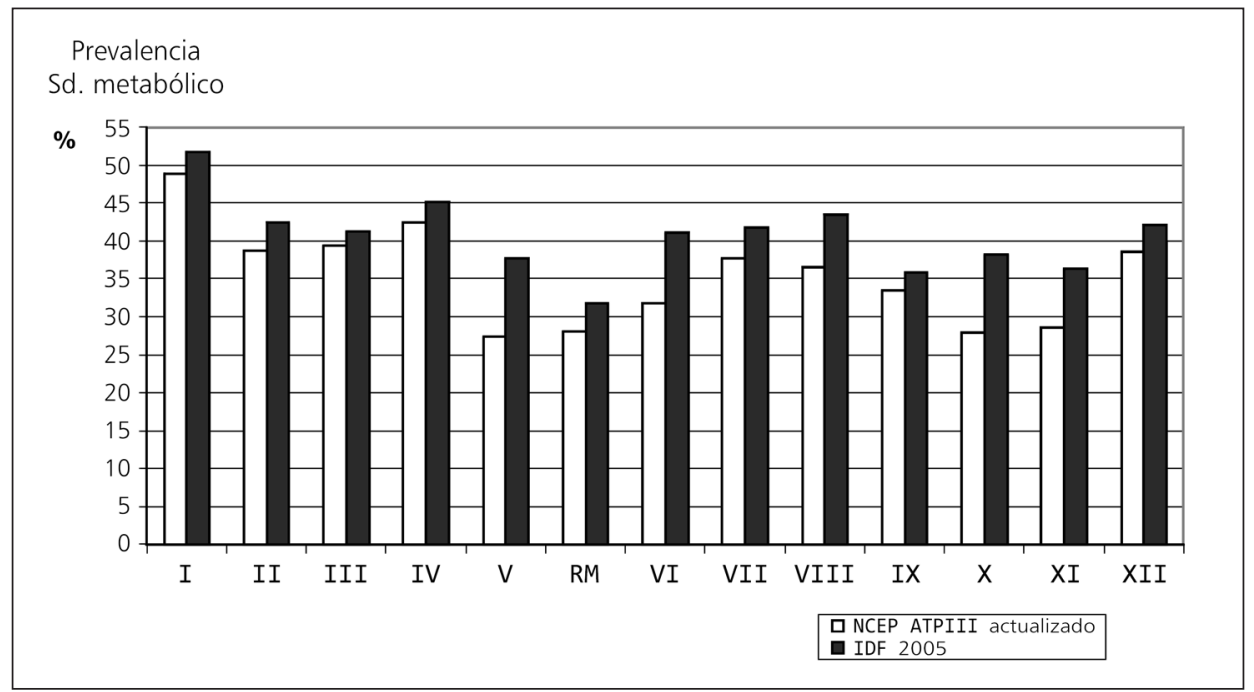

Figura 2. Prevalencia del síndrome metabólico según criterio ATP III actualizado e IDF en las diferentes Regiones de Chile.

género y edad para ambos criterios de diagnóstico. Las demás regiones no tuvieron diferencias significativas de prevalencia de SM con respecto a RM (Figura 2).

Finalmente, se exploró la asociación entre $\mathrm{SM}$ y sus factores de riesgo en Chile mediante un análisis transversal. Al analizar la prevalencia de SM según criterio ATPIII actualizado en obesos vs normopeso, se encontró que los obesos tienen aproximadamente 10 veces mayor prevalencia de SM que los normopeso, OR 9,59 (6,75-13,61). En sedentarios vs no sedentarios, se encontró que los sedentarios tienen el doble de SM, OR: 2,14 $(1,26-3,65)$. Estas asociaciones son significativas e independientes de la edad, género y nivel educa- cional de la población (Tabla 4). La prevalencia de personas que presentan simultáneamente sobrepeso y sedentarismo alcanzó a 55\%. El antecedente familiar de diabetes en la madre o el padre de un individuo fue significativamente mayor en personas con SM en ambas clasificaciones y presenta una significación límite cuando se ajustan por el resto de las variables.

\section{Discusión}

En nuestro trabajo se destaca la gran concordancia de la prevalencia de SM al ser definido con los criterios ATPIII actualizado e IDF. Ello difiere de lo publicado en otros países. Los datos de se- 
Prevalencia de síndrome metabólico en adultos chilenos - A. Valenzuela B. et al

Tabla 4. Comorbilidades asociadas al síndrome metabólico en población adulta, Chile, ENS 2003

\begin{tabular}{|c|c|c|c|}
\hline \multicolumn{4}{|c|}{ Prevalencia síndrome metabólico (ATP III actualizado) } \\
\hline & $\begin{array}{c}\text { No } \\
\text { (IC 95\%) }\end{array}$ & $\begin{array}{c}\text { Si } \\
\text { (IC 95\%) }\end{array}$ & $\begin{array}{l}\text { OR ajustado } \\
\text { (IC 95\%) }\end{array}$ \\
\hline Obesidad (IMC $\geq 30$ ) & $12,0 \quad(9,7-14,6)$ & $57,2(51,2-63,1)$ & $9,59(6,75-13,61)$ \\
\hline Sedentarismo & $85,7 \quad(81,6-89,1)$ & $93,8(90,6-96,0)$ & $2,1 \quad(1,26-3,65)$ \\
\hline
\end{tabular}

Regresión Logística, ajustada por edad, sexo, nivel educacional.

ries asiáticas muestran prevalencias de SM muy discordantes, lo que puede deberse a las diferentes características de la población. En el estudio transversal de Singapur, las prevalencias según ATPIII 2001 variaron en los 3 principales grupos étnicos: $9 \%$ chinos, $19 \%$ malayos y $20 \%$ indios asiáticos ${ }^{24}$. Otro estudio epidemiológico realizado en indios asiáticos, señaló diferencias de $18 \%$ y $26 \%$, ATP III 2001 e IDF respectivamente ${ }^{25}$, ello sugiere que utilizar los mismos criterios diagnósticos para toda Asia constituye un error metodológico.

Otras comparaciones entre los criterios diagnósticos para SM han sido recientemente realizadas en varias poblaciones. Lorenzo, Serrano y $\operatorname{cols}^{26}$ compararon la prevalencia de SM diagnosticado con los criterios ATPIII actualizado e IDF en diferentes grupos: Estados Unidos (USA) (San Antonio Texas, Mexicanos Americanos y blancos no hispánicos), México, España y Perú. Este estudio mostró diferencias en la prevalencia de SM entre los 2 criterios, las cuales fueron más significativas en hombres de México y Perú que en hombres de USA y España. Ambos criterios discreparon en la clasificación de SM en 13 a 29\% en hombres y en $3 \%$ a $7 \%$ en mujeres en ese estudio. Ello fue atribuido al uso de un criterio de CC para el diagnóstico de OA uniforme, que no corresponde a las distintas realidades.

Recientemente Assmann, Guerra y cols ${ }^{27} \mathrm{pu}-$ blicaron un trabajo que comparó en 3 muestras poblacionales, 2 de USA (Dallas Heart Study y NHANES 1999-2000) y 1 alemana (PROCAM), la prevalencia de SM diagnosticada por los criterios ATPIII actualizado e IDF. Cuando el criterio ATPIII actualizado fue usado, la prevalencia de SM en las muestras poblacionales USA fue mayor que en la muestra alemana, tanto para mujeres ( $37 \%$ vs $18 \%$ ) como para hombres ( $30 \%$ vs $25 \%$ ). Cuando el criterio de IDF fue usado, la prevalencia de SM fue $25 \%$ más alta en la muestra poblacional alema- na que en las muestras USA. Las diferencias en las prevalencias entre USA y Europa se relacionaron a los diferentes criterios de OA tanto para ATPIII actualizado como para IDF. Otro relevante aspecto de este trabajo fue la concordancia del diagnóstico entre las definiciones ATP III actualizado e IDF: $90 \%$ de concordancia para las 2 muestras poblacionales de USA, y un poco menor, $80 \%$ para la muestra alemana. En Chile, la concordancia del diagnóstico entre las definiciones ATP III actualizado e IDF fue 90\% (IC 95\% 88,1-91,7) similar a la muestra USA.

En nuestro país, la distribución de la frecuencia de los componentes individuales del SM: HTA, cHDL bajo e hipertrigliceridemia son altas y coexistentes, lo que puede explicar el buen nivel de concordancia entre ambos criterios del diagnóstico de SM. La OA si bien está presente, constituye el cuarto componente en una gran proporción de casos. El 27\% de la población chilena adulta presenta SM definido por 3 factores que no son OA.

Cabe destacar que el criterio ATPIII actualizado menciona que la CC debe ser medida en el borde superior de la cresta ilíaca en un plano paralelo al piso $^{13,17}$. En nuestro estudio la CC fue medida en la línea media axilar en el punto medio entre el reborde costal y la cresta ilíaca, ya que el estudio de Lean y $\mathrm{Han}^{14}$ (estudio que definió los pCC en base IMC) destaca la medida de la CC en este punto. Esta diferencia metodológica puede restar validez a la comparación entre ambas definiciones, subestimando la prevalencia de SM acorde al criterio ATPIII actualizado.

ES Ford ${ }^{28}$ estudió la prevalencia de SM en la ENS de los USA en adultos mayores de 20 años, demostrando que la prevalencia de SM aumenta de forma paralela con la edad. En base al criterio NCEP ATP III 2001, en hombres entre 30 y 39 años la prevalencia de SM fue $23 \%$, la que aumentó a $52 \%$ entre 60 a 69 años. En Chile ocurrió algo 
similar, en hombres entre 25 y 44 años la prevalencia de SM fue 31,1\% (IC 95\% 24,3-38,7), la que incrementó a $55,1 \%$ (IC 95\% 44,6-65,2) en hombres mayores de 65 años.

Los pCC sugeridos por IDF parecen sobrestimar la prevalencia de OA en nuestro país por lo que se deben definir en base a estudios locales.

Por el momento, independiente del criterio diagnóstico utilizado, la prevalencia de SM en Chile es alta y comparable a la de países desarrollados como USA, 55\% de los adultos chilenos presentan los principales detonantes del SM simultáneamente (sedentarismo y sobrepeso). Existe una fuerte asociación entre Obesidad-SM y Sedentarismo$\mathrm{SM}$, para ambos criterios del diagnóstico de SM en nuestra población (Tabla 4). Estos hechos, harán impracticable financiar un abordaje del problema por la vía de la atención médica individual, por lo que se necesitan paralelamente estrategias de intervención poblacional que se traduzcan en políticas de salud pública. Es necesario desplazar las curvas de distribución poblacional del sobrepeso y sedentarismo como una forma de disminuir la prevalencia de SM y sus consecuencias a largo plazo. Esperamos que este trabajo sea el inicio de una línea de estudio y perfeccionamiento de métodos diagnósticos para SM como una forma de aportar a la prevención de las enfermedades crónicas en nuestro país.

Agradecimientos: La Encuesta Nacional de Salud (ENS) 2003 fue financiada por la unidad de estudios y departamento de Epidemiología MINSAL (Dras. Ximena Aguilera, Claudia González y equipo). Los subestudios relacionados con SM han sido apoyados posteriormente con aportes del Proyecto FONDECYT Regular No 1070634, Dr. Atilio Rigotti.

\section{Referencias}

1. Reaven G. Role of insulin resistance in human disease. Diabetes 1988; 37: 1495-607.

2. Grundy SM, Brewer HB Jr, Cleeman JI, Smith SC Jr, Lenfant C. American Heart Association; National Heart, Lung, and Blood Institute. Definition of metabolic syndrome: report of the National Heart, Lung, and Blood Institute/American Heart Association conference on scientific issues related to definition. Circulation 2004; 109: 433-8.

3. Stern MP, Williams K, González-Villalpando C, Hunt
KJ, Haffner SM. Does the metabolic syndrome improve identification of individuals at risk of type 2 diabetes and/or cardiovascular disease? Diabetes Care 2004; 27: 2676-81.

4. Hanson RL, Imperatore G, Bennett PH, Knowler WC. Components of the "metabolic syndrome" and incidence of type 2 diabetes. Diabetes 2002; 51: 3120-7.

5. Lemieux I, Pascot A, Couillard C, Lamarche B, Tchernof A, Almeras N, et al. Hypertriglyceridemic waist: a marker of the atherogenic metabolic triad (hyperinsulinemia; hyperapolipoprotein B; small, dense LDL) in men? Circulation 2000; 102: 179-84.

6. Carr DB, Utzschneider KM, Hull RL, Kodama K, Retzlaff $\mathrm{BM}$, Brunzell JD, et al. Intra-abdominal fat is a major determinant of the National Cholesterol Education Program Adult Treatment Panel III criteria for the metabolic syndrome. Diabetes 2004; 53: 2087-94.

7. Reaven GM. The metabolic syndrome: requiescat in pace. Clin Chem 2005; 51: 931-8.

8. Alberti KGMM, Zimmet P, Shaw J. Metabolic SyndromeA new world-wide definition. A consensus statement from the International Diabetes Federation. Diabet Med 2006; 23: 469-80.

9. Ford ES. Risks for all-cause mortality, cardiovascular disease, and diabetes associated with the metabolic syndrome. Diabetes Care 2005; 28: 1769-78.

10. Hanley A, Karter A, Williams K, Festa A, D’Agostino $\mathrm{R}$, Wagenknecht L, et al. Prediction of type 2 diabetes mellitus with alternative definitions of the metabolic syndrome: the Insulin Resistance Atherosclerosis Study. Circulation 2005; 112: 3713-21.

11. Executive Summary of The Third Report of The National Cholesterol Education Program (NCEP) Expert Panel on Detection, Evaluation, and Treatment of High Blood Cholesterol In Adults (Adult Treatment Panel III). JAMA 2001; 285: 2486-97.

12. WHO consultation: Definition, diagnosis and classification of diabetes mellitus and its complications. WHO/ NCD/NCS/99.2; 31-3.

13. Clinical Guidelines on the Identification, Evaluation, and Treatment of Overweight and Obesity in Adults-the Evidence Report. National Institutes of Health. Obes Res. 1998; 6 (suppl 2): 51S-209S.

14. Lean ME, Han TS, Morrison CE. Waist circumference indicates the need for weight management. BMJ 1995; 311: 158-61.

15. Han TS, Van Leer EM, Seidell JC, et al. Waist circumference action levels in the identification of cardiovascular risk factors: prevalence study in a random sample. BMJ 1995; 311: 1401-5.

16. Laaksonen D, Lakka H, Niskanen L, Kaplan G, Salonen J, 
Prevalencia de síndrome metabólico en adultos chilenos - A. Valenzuela B. et al

Lakka T. Metabolic Syndrome and Development of Diabetes Mellitus: Application and Validation of Recently Suggested Definitions of the Metabolic Syndrome in a Prospective Cohort Study. Am J Epidemiol 2002; 156: 1070-7.

17. Grundy SM, Cleeman JI, Daniels SR, Donato KA, Eckel RH, Franklin BA, et al. Diagnosis and management of the metabolic syndrome. An American Heart Association/National Heart, Lung, and Blood Institute Scientific Statement. Circulation 2005; 112: 2735-52.

18. Genuth S, Alberti KG, Bennett P, Buse J, Defronzo R, Kahn R, et al. Expert Committee on the Diagnosis and Classification of Diabetes Mellitus. Follow-up report on the diagnosis of diabetes mellitus. Diabetes Care 2003; 26: 3160-7.

19. International Diabetes Federation: Worldwide definition of the metabolic syndrome. Available at: http://www.idf. org/webdata/docs/IDF_Meta-syndrome_definition.pdf. Accessed August 24, 2005.

20. Alberti KG, Zimmet P, Shaw J. Metabolic syndrome-a new world-wide definition. A Consensus Statement from the International Diabetes Federation. Diabet Med 2006; 23: 469-80.

21. Minsal. Encuesta Nacional de Salud Chile, 2003.

22. http://epi.minsal.cl/epi/html/invest/ENS/InformeFinalENS.pdf.

23. Landis JR, Koch GG. The measurement of observer agreement for categorical data. Biometrics 1977; 33: 159-74.

24. Tan CE, Ma S, Wai D, Chew S, Shyong E. Can we apply the National Cholesterol Education Program Adult Treatment Panel definition of the metabolic syndrome to Asians? Diabetes Care 2004; 27: 1182-6.

25. Deepa M, Farooq S, Datta M, Deepa R, Mohan V. Prevalence of metabolic syndrome using WHO, ATPIII and IDF definitions in Asian Indians: The Chennai Urban Rural Epidemiology Study (CURES-34). Diabetes Metab Res Rev 2007; 23: 127-34.

26. Lorenzo C, Serrano-Ríos M, Martínez-Larrad M, González-Sánchez J, Seclén S, Villena A, et al. Geographic variations of the International Diabetes Federation and the National Cholesterol Education Program-Adult Treatment Panel III definitions of the metabolic syndrome in nondiabetic subjects.Diabetes Care 2006; 29: 685-91.

27. Assmann G, Guerra R, Fox G, Cullen P, Schulte H, Willett D. Harmonizing the definition of the metabolic syndrome: comparison of the criteria of the Adult Treatment Panel III and the International Diabetes Federation in United States American and European populations. Am J Cardiol 2007; 99: 541-8.

28. Ford ES. Prevalence of the Metabolic Syndrome Defined by the International Diabetes Federation Among Adults in the US. Diabetes Care 2005; 28: 2745-9. 